MODELING, IDENTIFICATION AND CONTROL, 1983, vOL. 4, NO. 2, 63-81

doi:10.4173/mic.1983.21

\title{
An adaptive multivariable control system for hydroelectric generating units $\dagger$
}

\author{
GUNNE JOHN HEGGLID $\ddagger$
}

Keywords: adaptive control, multivariable systems, hydroelectric generating units, Kalman filtering.

\begin{abstract}
This paper describes an adaptive multivariable control system for hydroelectric generating units. The system is based on a detailed mathematical model of the synchronous generator, the water turbine, the exiter system and turbine control servo. The models of the water penstock and the connected power system are static. These assumptions are not considered crucial. The system uses a Kalman filter for optimal estimation of the state variables and the parameters of the electric grid equivalent. The multivariable control law is computed from a Riccatti equation and is made adaptive to the generators running condition by means of a least square technique.
\end{abstract}

\section{Introduction}

Successful operation of a power system depends largely on the ability to provide reliable and uninterrupted service. The reliability of the power supply implies much more than merely being available. Ideally, the loads must be fed at constant voltage and frequency at all times. In practical terms this means that both voltage and frequency must be held within close tolerances so that the consumer's equipment may operate satisfactorily. For example, a drop in voltage of $10-15 \%$ or a reduction of the system frequency of only a few hertz may lead to stalling of the motor loads on the system.

The first requirement of reliable service is to keep the generators running in parallel and with adequate capacity to meet the load demand. If at any time a generator loses synchronism with the rest of the system, significant voltage and current fluctuations may occur and transmission lines may be automatically tripped by their relays at undesired locations. If a generator is separated from the system it must be resynchronized and then loaded, assuming it has not been damaged and its turbine has not been shut down due to the disturbance that caused the loss of synchronism.

A second requirement of reliable electrical service is to maintain the integrity of the power network. The high-voltage transmission system connects the generating stations and the load centres. Interruptions in this network may hinder the flow of power to the load. This usually requires a study of large geographical areas since almost all power stations and load centres are connected in one power system.

This paper presents a design method for an optimal controller for hydroelectric generating units based on state and parameter estimation by an augmented Kalman filter. The controller is to maintain both the requirements; keeping the generators running in parallel and to maintain the integrity of the power system. This is done

Received 17 August 1982.

† This work was done as the author had a scholarship from the University of Trondheim, The Norwegian Institute of Technology, N-7034 Trondheim-NTH, Norway.

† IGP A/S., Dronningens gt 7, N-7000 Trondheim, Norway.

M.I.C. 
mainly by reducing the first swing of the rotors of the synchronous generators after large disturbances and to increase the damping of power oscillations.

\section{Mathematical modeling}

The mathematical model is designed to be used in a Kalman filter for the estimation of the state variables and the parameters of the generator, the water turbine and the connected electric grid. The model will consist of a third order model for the synchronous generator and a first order model for the turbine control servo. The power system model is a static equivalent, and the water penstock is modeled as a white noise process.

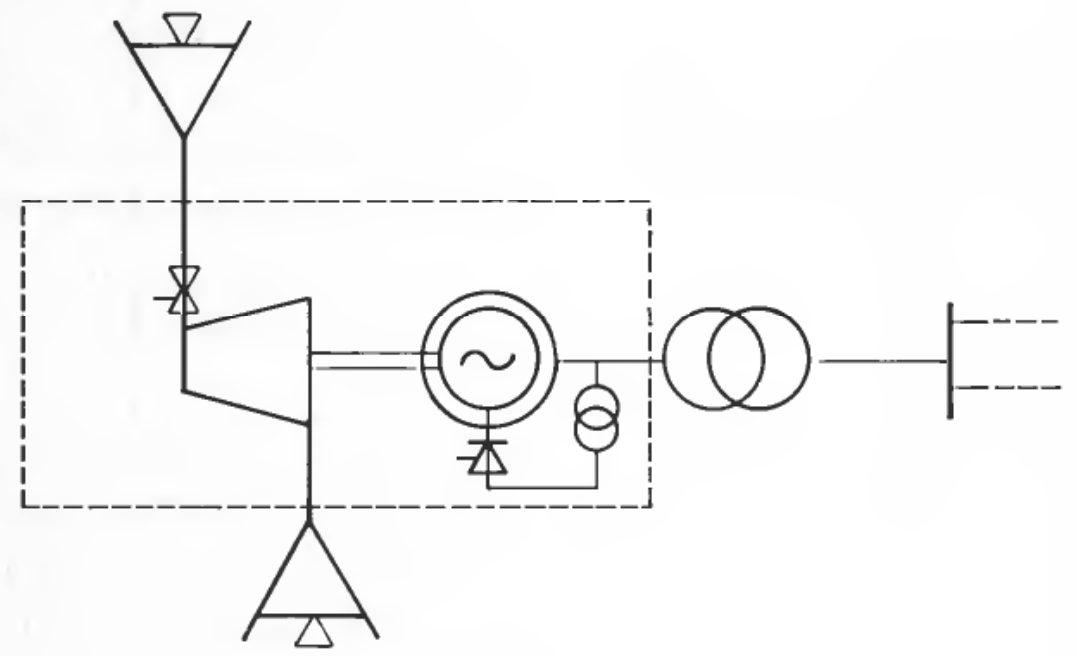

Figure 1. A hydroelectric generating unit.

The following symbols are used:

$\gamma \quad$ angle between $q$-axis and external equivalent voltage

$\omega_{0} \quad=2 \pi f=100 \pi$

$\omega_{g} \quad$ mechanical speed

$\omega \quad$ speed of external equivalent voltage

$m_{t} \quad$ water turbine torque

$m \delta \quad$ electrical torque

$D$ damping constant

$T_{a} \quad$ mechanical time constant

$E_{q}^{\prime} \quad$ field flux equivalent voltage

$E_{a f} \quad$ field voltage

$x_{d} \quad d$-axis synchronous reactance

$x_{d}^{\prime} \quad$ transient $d$-axis reactance

$x_{q} \quad q$-axis synchronous reactance

$I_{d} \quad d$-axis component of generator current

$I_{q} \quad q$-axis component of generator current

$K_{E} \quad$ thyristor bridge gain

$U$ external equivalent voltage 
$r$ network equivalent resistance

$x$ network equivalent reactance

$\eta^{\prime} \quad$ turbine efficiency

$q_{t} \quad$ turbine water flow

$h$ turbine inlet pressure

$y$ prime mover position

$K_{y} \quad$ prime mover gain

$T_{y} \quad$ prime mover time constant

$u_{1} \quad$ control signal to prime mover

$u_{2} \quad$ control signal to thyristor bridge

$U_{g} \quad$ generator voltage

$I_{g} \quad$ generator current

$\boldsymbol{P}_{g} \quad$ generator active power output

$Q_{g} \quad$ generator reactive power output

$v_{h} \quad$ white noise on inlet pressure $h$

$v_{r} \quad$ white noise on resistance $r$

$v_{x} \quad$ white noise on reactance $x$

$v_{u} \quad$ white noise on voltage $U$

$v_{\omega} \quad$ white noise on speed

$\lambda_{\gamma}$ gain factor for speed/prime mover position

$\lambda_{p} \quad$ gain factor for speed/active power

$Q_{n} \quad$ reactive power output from transformer

$V_{n} \quad$ voltage on network side of transformer

$X_{c} \quad$ compensating reactance

\subsection{The generator model}

Figure 2 shows a phasor diagram for the synchronous machine connected to a constant voltage through an impedance. The dynamic behaviour of the generator is described by the following equations, (1)-(4).

$$
\begin{aligned}
\dot{\gamma} & =\omega_{0}\left(\omega_{g}-\omega\right) \\
\dot{\omega}_{g} & =\left(m_{t}-m_{\delta}-D\left(\omega_{g}-\omega\right)\right) / T_{a} \\
\dot{E}_{q}^{\prime} & =\left(E_{q f}-E_{q}^{\prime}-\left(x_{d}-x_{d}^{\prime}\right) I_{d}\right) / T_{d 0}^{\prime} \\
m_{\delta} & =\left(E_{q}^{\prime}+\left(x_{q}-x_{d}^{\prime}\right) I_{d}\right) I_{q}
\end{aligned}
$$

Equation (1) describes the motion of the angle between the machine rotor and the voltage of the external equivalent, $(2)$ is the swing equation which governs the generator speed relating the inertia torque to the resultant of the mechanical and electrical torques on the rotor, (3) describes the variation of the field flux-linkages, (4) shows the calculation of the electrical torque.

\subsection{The exitation system}

Since the late 1960 s almost all water power generators in Norway are built without DC-generator(s) for exitation. The rotor winding is fed through a three phase thyristor bridge as shown in Fig. 1,

When neglecting the thyristor bridge time constant (approx. $0.01 \mathrm{sec}$ ), the mathematical model of the exitation system is

$$
E_{a f}=K_{E} u_{2}
$$




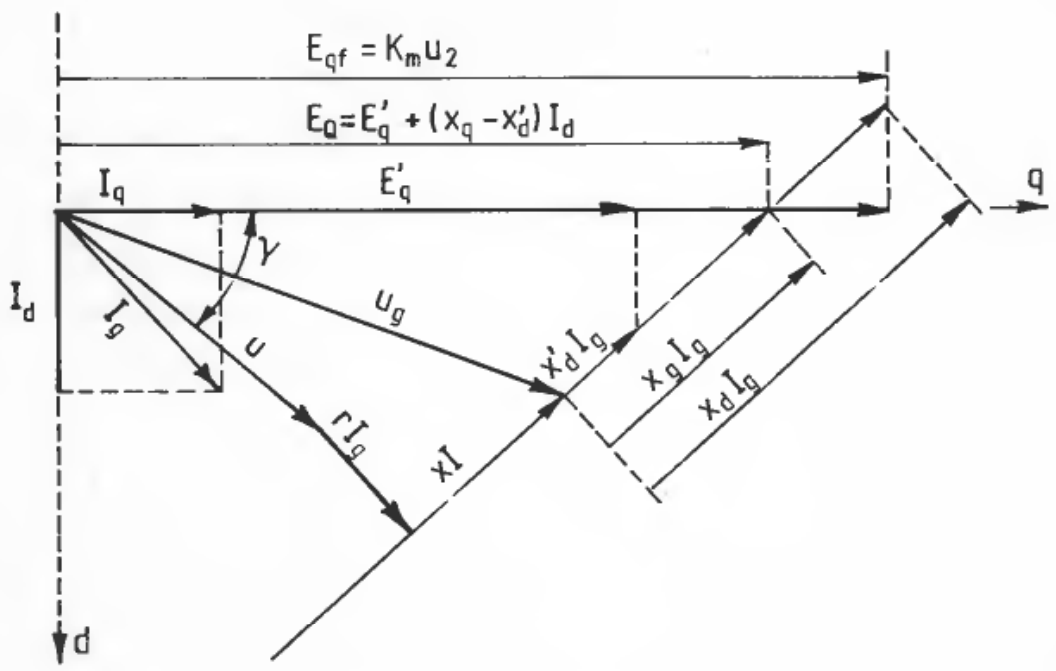

Figure 2. The phasor diagram for the generator and the grid equivalent.

\subsection{The grid model}

The generating unit which we want to control is connected to a power system. This may consist of several other generating units, transmission lines, transformers and load centres. It would be a gigantic mathematical model if we modeled it all in detail. We therefore make a dramatic simplification and suppose the external grid to be a static equivalent as shown in Fig. 2. The equivalent consists of a 'constant' impedance $(r+j x)$, a 'constant' voltage $(U)$, with 'constant' frequency $(\omega)$.

$$
\begin{aligned}
& I_{d}=\frac{1}{x_{1}}\left(E_{q}^{\prime}-U \cos \gamma-\frac{r}{x_{2}} U \sin \gamma\right) \\
& I_{q}=\frac{1}{x_{2}}\left(\frac{r}{x_{1}} E_{q}^{\prime}-\frac{r}{x_{1}} U \cos \gamma+\left(1-\frac{r^{2}}{x_{1} x_{2}}\right) U \sin \gamma\right) \\
& x_{1}=x+x_{d}^{\prime}+\frac{r_{2}}{x_{2}} \\
& x_{2}=x+x_{q}
\end{aligned}
$$

\subsection{The water penstock and turbine model}

Different mathematical models have been developed to account for the effect of the water turbine and penstock. Most of the turbine models are static, but the water in the penstock is modeled dynamically in different ways. Here we want to describe the 
turbine to be a static element for energy conversion and the penstock, by the pressure at the turbine inlet, to be a white noise process.

$$
\begin{gathered}
m_{t}=\eta^{\prime} q_{t} h / \omega_{g} \\
h=0+v_{h}
\end{gathered}
$$

$\eta^{\prime}$ the turbine efficiency, is found from the turbine's characteristic diagram and depends on both the rotational speed $\omega_{g}$, the pressure $h$, and the turbine flow $q_{t}$ (Appendix and Hegglid, 1981).

The most economical layout of a high head type power plant consists of an unlined sloping headrace tunnel ending in a short lined penstock into the power house, and an air accumulator or a surge shaft to damp oscillations in the upstream tunnel system. The damping of hydraulic oscillations due to prime mover movements in most unlined tunnels and shafts is significant compared to lined systems (Brekke and Bugaarden, 1980). In addition, in Hegglid (1981) it is shown that the contribution from the water turbine and prime mover to the damping of power system oscillation is much less than the contribution from the exitation system. Therefore the use of the prime mover control is reduced by differences in the weighting matrix $P$ (see $\$ \S 4$ and 5). Hence, the water penstock model is considered to be a white noise process which will 'follow' the mean value variations of the pressure at the turbine inlet, and therefore be an important input to the calculation of the turbine efficiency.

From this the assumption about the static penstock model is considered not to be crucial. But if it is considered necessary to include a dynamic model for the hydraulic headrace tunnel system this does not change the principles of the control system, although the power system damping may be increased.

\subsection{The turbine control servo}

The prime mover of the water turbine is the second control input of the generating unit. It consists of an electro-hydraulic amplifier. A sufficient mathematical model for the main servo is one time constant.

$$
\dot{y}=\left(K_{y} u_{1}-y\right) / T_{y}
$$

\subsection{The state space model}

Equations (1)-(10) form an unlinear, fifth order, state space model.

$$
\boldsymbol{x}=\left(\gamma, \omega_{g}, E_{q}^{\prime}, y, h\right)^{T}
$$

The feedback from the optimal control law is based on $x$. This vector is estimated through a Kalman filter augmented with the electric grid parameters $r, x, U$ and $\omega$.

$$
\tilde{\boldsymbol{x}}=\left(x^{\prime}, r, x, U, \omega\right)^{T}
$$

The measurements of the generating unit are

$$
\boldsymbol{y}=\left(\omega_{g}, U_{g}, I_{g}, P_{g}, Q_{g}, y, h\right)^{T}
$$


The measurement vector is given through non-linear functions from the state space vector $\tilde{\boldsymbol{x}}$.

$$
\begin{aligned}
\omega_{g} & =\omega_{g} \\
U_{g} & =\left(U_{d}{ }^{2}+U_{q}{ }^{2}\right)^{1 / 2} \\
I_{g} & =\left(I_{d}{ }^{2}+I_{q}{ }^{2}\right)^{1 / 2} \\
P_{g} & =U_{d} I_{d}+U_{q} I_{q} \\
Q_{g} & =U_{q} I_{d}-U_{d} I_{q} \\
y & =y \\
h & =h \\
U_{d} & =x_{q} I_{q} \\
U_{q} & =E_{q}^{\prime}-x_{d}^{\prime} I_{d}
\end{aligned}
$$

For the estimator we want to use the non-linear equations (1)-(10) and (14)-(22). But the optimal controller theory is based on a linear state space model. Therefore also a linear state space model should be derived. The vectors of the state space model can be expressed as a deviation from a working point.

$$
\begin{aligned}
\boldsymbol{x} & =\boldsymbol{x}_{0}+\delta \boldsymbol{x} \\
\boldsymbol{y} & =\boldsymbol{y}_{0}+\delta \boldsymbol{y} \\
\boldsymbol{i} & =\boldsymbol{i}_{0}+\delta \boldsymbol{i} \\
\boldsymbol{u} & =\boldsymbol{u}_{0}+\delta \boldsymbol{u} \\
\boldsymbol{i} & =\left(I_{d}, I_{q}\right)^{T} \\
\boldsymbol{u} & =\left(u_{1}, u_{2}\right)^{T} \\
\delta \dot{\boldsymbol{x}} & =A^{\prime} \delta \boldsymbol{x}+E \delta \boldsymbol{i}+B \delta \boldsymbol{u} \\
\delta \boldsymbol{i} & =H \delta \boldsymbol{x} \\
\delta \boldsymbol{y} & =D^{\prime} \delta \boldsymbol{x}+F \delta \boldsymbol{i}
\end{aligned}
$$

The matrix $H$ depencis on the external grid parameters, $r, x$, and $U$, and the system is therefore time variant. Using eqn. (30) in (29) and (31) results in

$$
\begin{aligned}
& \delta \dot{x}=A \delta x+B \delta u \\
& \delta y=D \delta x
\end{aligned}
$$

\section{The estimator system}

The estimation of states and parameters is based on the non-linear model previously shown. The external grid parameters $r, x, U$ and $\omega$ are modeled as white noise processes.

$$
\begin{aligned}
& \dot{r}=v_{r} \\
& \dot{x}=v_{x} \\
& \dot{u}=v_{u} \\
& \dot{\omega}=v_{w}
\end{aligned}
$$




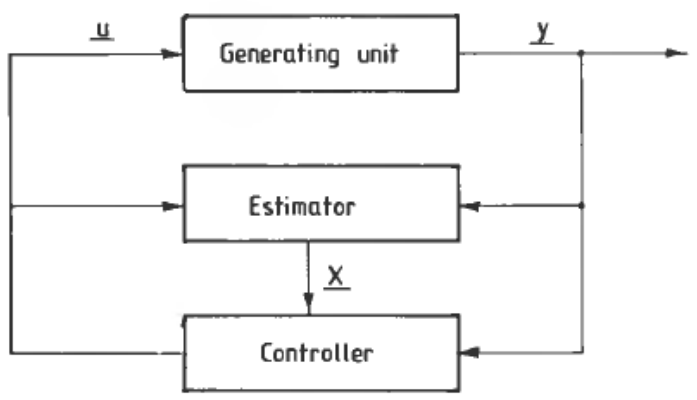

Figure 3. Generating unit with controller. The controller system contains both working point and optimal controller.

The total control system with augmented Kalman filter is shown in Fig. 3. The Kalman filter gain $K(t)$ is calculated from the covariance matrices $V$ and $W$. $W$, the measurement noise covariance is supposed to be diagonal and its elements given by the variances of the measurement errors. The process noise, described by $V$, contains noise on the measurements of the control input signal, the incoming disturbances from the connected power system and the uncertainties in the modeling of the generating unit and power system. The $V$-matrix is also supposed to be diagonal. Its elements are found after the eigenvalues of the control loops are fixed. This is discussed later. Both $V$ and $W$ are supposed to be time invariant.

\section{The control system}

The control system contains two parts: a slow working point controller and a fast acting optimal controller.

\subsection{The working point controller}

The main task of the working point controller is to make the average performance of the electric power generating unit follow given references. To be able to follow the references with small deviations is a matter of considerable importance both to system economics and system operation. To obtain a minimum of interaction between the working point controller and the fast acting optimal controller the working point controller is acting on the working points, see eqns. (23)-(26). The working point controller can also be divided into two parts; the control of active power and rotational speed, frequency, and the control of generator voltage and reactive power.

\subsubsection{The active power - controlling frequency}

It is necessary for all the generators of the interconnected power system to run stable in parallel. This is achieved by keeping a negative characteristic between the rotational speed and the output of active power, $\boldsymbol{P}_{g}$, from the generator.

This negative characteristic is obtained by the following

$$
y=y_{\text {ref }}-\lambda_{f}\left(\bar{\omega}_{g}-\omega_{\text {ref }}\right)
$$

The active power $\boldsymbol{P}_{g}$, is stationary proportional to the position of the prime mover of the water turbine, $y$.

$$
P_{g} \triangleq y
$$


Therefore

$$
P_{g}=P_{\text {ref }}-\lambda_{p}\left(\bar{\omega}_{g}-\omega_{\text {ref }}\right)
$$

The reference of active power is given by a higher level of (economic) control and the reference of rotational speed is system nominal frequency. The factor $\lambda$ is a frequency amplification factor and is also given from considerations on system economics and operation.

\subsubsection{The voltage - controlling reactive power}

Most modern generating units are connected to the high voltage transmission system by separate transformers (see Fig. 4).
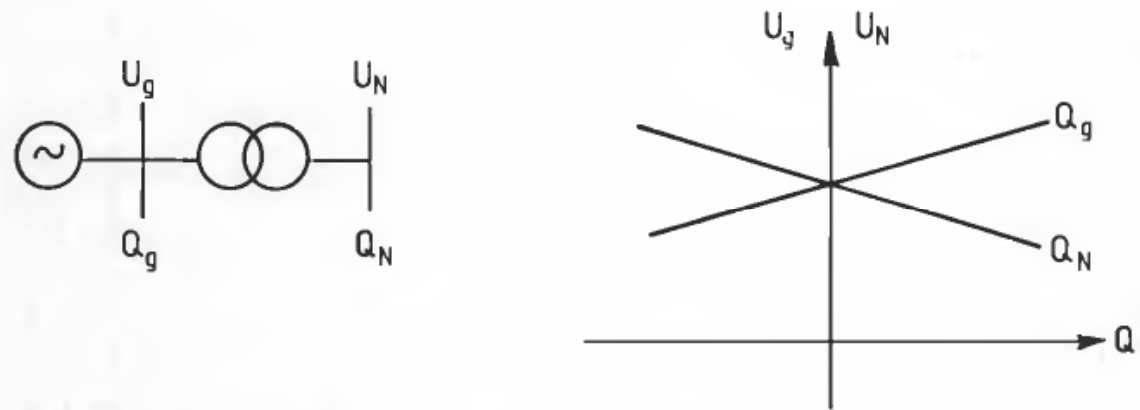

Figure 4. Generator and transformer. Characteristics for the variation of voltages on generator and network busbar from variation of generator reactive power output.

Both for stabilized output of reactive power from generators in parallel and minimum variation of network voltage $U_{g}$, the working point controller for generator voltage is compensated for variation of reactive power output, $Q_{g}$ :

$$
U_{g}-x_{c} \frac{Q_{g}}{U_{g}}=U_{\text {rer }}
$$

$x_{c}$, the compensating reactance is given from the point at which we want to keep constant voltage.

\subsubsection{The working point controller equations}

As mentioned, the working point controller is acting on the base of the calculation of deviations in estimated states, the working point vector, $x_{0}$. Section 4.1.2 shows the references of the generating units' performance. The following will show how the elements of $x_{0}$ are calculated.

$$
\begin{aligned}
x_{0} & =\left(\gamma_{0}, \omega_{a 0}, E_{a 0}^{\prime}, y_{0}, h_{0}\right)^{T} \\
\omega_{g 0} & =\bar{\omega}_{g} \\
h_{0} & =h_{0} \\
y_{0} & =y_{\text {ref }}-\lambda_{y}\left(\bar{\omega}_{g}-\omega_{\text {ref }}\right) \\
P_{0} & =P_{\text {ref }}-\lambda_{p}\left(\bar{\omega}_{g}-\omega_{\text {ref }}\right) \\
U_{0} & =U_{\text {ref }}-x_{e} \frac{\bar{Q}_{g}}{U_{\text {ref }}}
\end{aligned}
$$


From the phasor diagram (Fig. 2) we can calculate $\gamma_{0}$, the angle between the $q$-axis and the external voltage $U$, and the voltage $E_{q}^{\prime}$.

$$
\begin{aligned}
I_{a 0} & =P_{0} / U_{0} \\
I_{r 0} & =-Q_{g} / U_{0} \\
\gamma_{0} & =\gamma_{1}+\gamma_{2} \\
\gamma_{1} & =a \tan \left(x_{q} I_{a 0} /\left(U_{0}-x_{q} I_{r 0}\right)\right) \\
\gamma_{2} & =a \tan \left(\left(x I_{a 0}+r I_{r 0}\right) /\left(U_{0}-r I_{a 0}+x I_{r 0}\right)\right) \\
I_{d 0} & =I_{a 0} \sin \gamma_{1}-I_{r 0} \cos \gamma_{1} \\
E_{q 0}^{\prime} & =\left(\left(U_{0}-x_{q} I_{r 0}\right)^{2}+\left(x_{q} I_{a 0}\right)^{2}\right)^{1 / 2}-\left(x_{q}-x_{d}^{\prime}\right) I_{d 0}
\end{aligned}
$$

\subsection{The optimal controller}

The output of the optimal controller is computed from the deviations of the estimated state variables from the working point $x_{0}$. The controller is based on a quadratic performance criterion and optimal control theory for linear systems. In order to find the controller feedback matrix $G$, the model is linearized around the working point as previously shown (see eqns (32)-(33)). To realize a controller on a computer the model must be converted to discrete time

$$
\begin{aligned}
\delta \boldsymbol{x}(k+1) & =\boldsymbol{\Phi} \delta \boldsymbol{x}(k)+\Delta \delta \boldsymbol{u}(k) \\
\delta \boldsymbol{y}(k) & =D \delta \boldsymbol{x}(k)
\end{aligned}
$$

$\Phi$ and $\Delta$ are found from series expansions. $T$ is the sampling time.

$$
\begin{aligned}
& \Phi \approx I+A T+\frac{1}{2} A^{2} T^{2} \\
& \Delta \approx B T
\end{aligned}
$$

The feedback matrix is found by minimizing the functional

$$
J=\frac{1}{2} \sum^{\infty}\left(\delta x(k)^{T} Q \delta x(k)+\delta u(k)^{T} P \delta u(k)\right) \quad k=0
$$

The matrix $P$, the weighting matrix for the controller outputs, is fixed. This matrix will define the comparative use of the two control outputs. $P$ is assumed to be diagonal. The matrix $Q$, the weighting matrix for state deviations, is determined from the closed loop dynamic performance, i.e. the position of the closed loop eigenvalues.

$$
\delta \boldsymbol{x}(k+1)=(\Phi+\Delta G) \dot{\delta} \boldsymbol{x}(k)
$$

Most attention was paid to damping of angle/speed oscillations.

\subsection{The eigenvalues of the estimator}

The determination of the eigenvalues of the controller $(\Phi+\Delta G)$ is shown in $\S 4.2$. The matrix $V$, the covariance of the process noise, is determining the eigenvalues of the estimator $(\Phi-K D)$. When the eigenvalues of the controller are found/decided, the dynamics of the estimator are determined to be faster than the dynamics of the controller. This is done by separating the eigenvalues of the estimator from the eigenvalues of the controller, by varying $V$. One example of eigenvalue location is shown in Fig. 5 . 


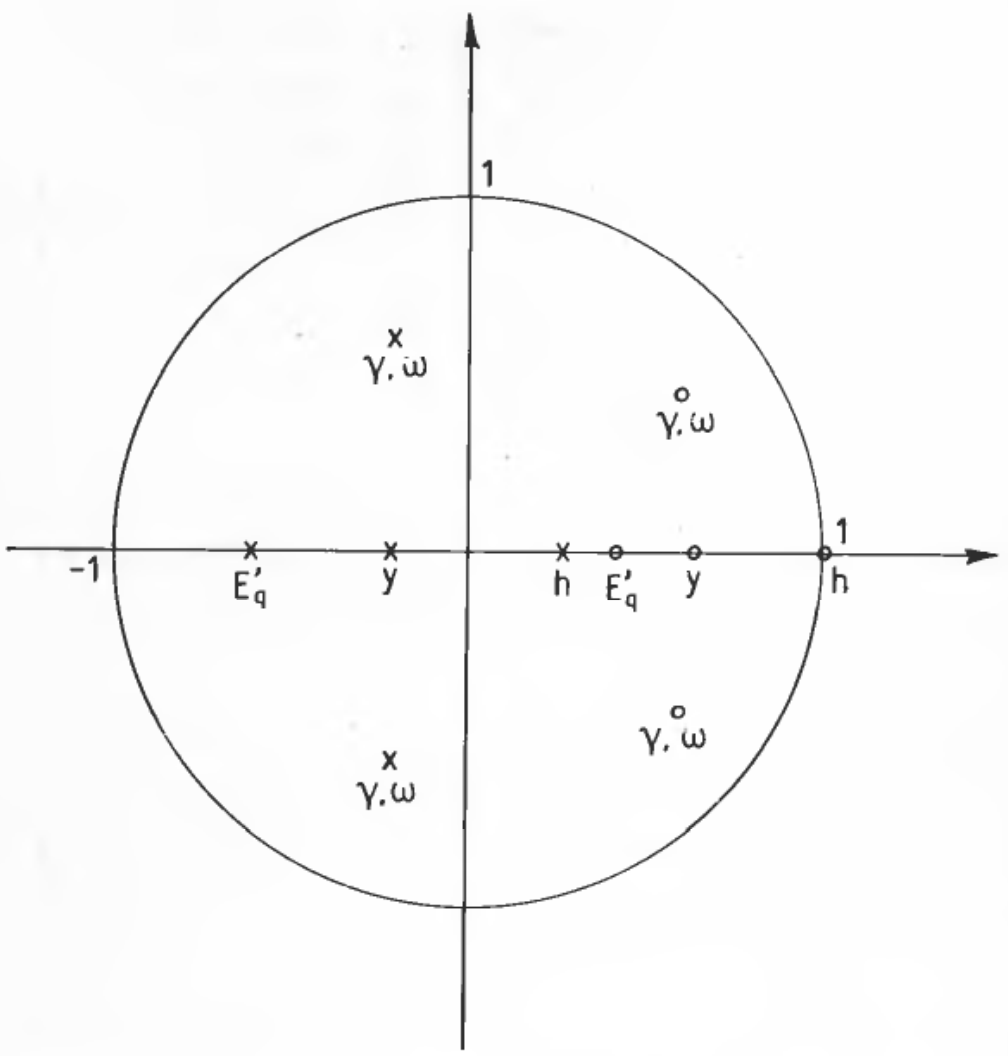

Figure 5. Eigenvalue location of estimator and controller: $\bigcirc$-eigenvalues of estimator: $x$-eigenvalues of controller.

\subsection{Adaptive optimal controller}

Because of changes in the power network breaker status, loading, voltages etc., together with variations in active and reactive output from the generating unit, the dynamics will change. This will cause changes in the closed loop eigenvalues, and the damping of angle oscillations may be decreased, and even become negative (unstable).

Therefore an algorithm for adapting the optimal controller to the variations in working conditions was suggested. We assume the optimal controller feedback to depend on generator voltage and active power output and resistance, reactance and voltage of the external grid equivalent.

$$
\begin{aligned}
G & =G\left(U_{g}, P_{g}, r, x, U\right) \\
G^{*} & =G_{0}{ }^{*}+G_{1} * \mu_{1}\left(U_{g}\right)+G_{2} * \mu_{2}\left(P_{g}\right)+G_{3} * \mu_{3}(r)+G_{4} * \mu_{4}(x)+G_{5} * \mu_{5}(U)
\end{aligned}
$$

The coefficient matrices $G^{*}$ are constant and found, when the functions $\mu i$ are given, by varying the working conditions and solving functional (49). In this paper the $P$ and $Q$ matrices are supposed to be constant and not dependent on the working condition. The matrices $G^{*}$ are determined by using a least squares method.

The resulting feedback, (51), is calculated from the working point and the estimates of $r, x$ and $U$. 


\section{Simulations}

For showing the properties of an adaptive optimal controller for hydroelectric generating units, some simulations on a power system simulator on a NORD 10 computer are carried out.

\subsection{Example network}

The power network used in the simulations carried out in this paper is shown in Fig. 6. The power system simulator contains a fifth order model for the synchronous generators, third order turbine servo and penstock model (including the 'waterhammer'). The controller system with estimator and controller is connected to the power system simulator only via 'measurements' on the actual 'generating unit' and the controller signals are 'inserted' into the generating units control inputs; prime mover and exiter inputs. Hence the models of the generating unit in the simulator and estimator are unequal as both generator, power system, turbine and penstock models are unequal.

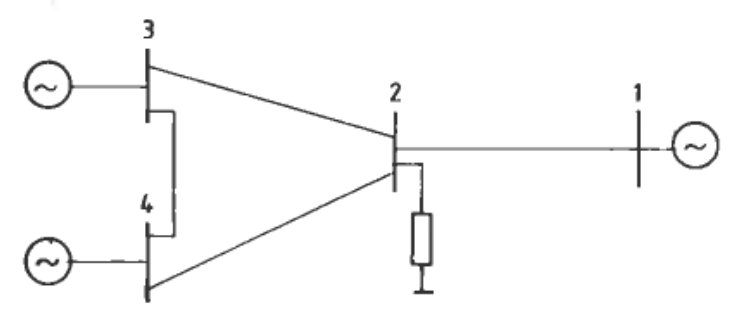

Figure 6. Example network (data shown in Appendix).

\subsection{Computation of the adaptive control law}

For one working condition of the actual generating unit the choice of the weighting matrices $P$ and $Q$ was done. This was done to obtain sufficient damping of angle oscillations.

$$
\begin{aligned}
& P=\operatorname{diag}(25,1) \\
& Q=\operatorname{diag}(1000,1000, \cdot 01,10,0)
\end{aligned}
$$

With the following functions $\mu$, the adaptive control law was determined.

$$
\begin{array}{ll}
\mu_{1}=U-1 \cdot 0 & \mu_{4}=x \\
\mu_{2}=\boldsymbol{P}-0.8 & \mu_{5}=\boldsymbol{U} \\
\mu_{3}=r &
\end{array}
$$

The determination was done by varying the working conditions.

$$
\begin{aligned}
& 0.8<U<1.2 \\
& 0.2<P<1.4 \\
& 0.0<r<1.0 \\
& 0.0<x<2.5 \\
& 0.8<U<1.2
\end{aligned}
$$




\begin{tabular}{lrrrrr}
\hline$G_{0}{ }^{*}$ & -0.008 & 0.272 & -0.002 & 0.010 & 0.007 \\
& -0.014 & -10.654 & 0.067 & -0.175 & -0.113 \\
\hline$G_{1}{ }^{*}$ & -0.013 & -0.833 & -0.009 & 0.029 & 0.022 \\
& -0.624 & -42.197 & 0.815 & -0.877 & -0.575 \\
\hline$G_{2}{ }^{*}$ & -0.017 & 0.426 & -0.001 & 0.020 & 0.015 \\
& -0.149 & -1.815 & -0.165 & -0.094 & -0.045 \\
\hline$G_{3}{ }^{*}$ & 0.001 & 0.025 & 0.004 & -0.025 & -0.018 \\
& -0.065 & 0.015 & -0.270 & 0.447 & 0.282 \\
\hline$G_{4}{ }^{*}$ & -0.006 & -0.099 & 0.013 & 0.000 & -0.009 \\
& 0.261 & 7.986 & -0.160 & 0.024 & 0.326 \\
\hline$G_{5}{ }^{*}$ & 0.002 & -0.069 & 0.001 & -0.002 & -0.002 \\
& -0.062 & 5.234 & -0.059 & 0.097 & 0.060 \\
\hline
\end{tabular}

Table 1. The adaptive control law.

5.3. The noise matrices $V$ and $W$, and the estimator gain

After determining the controller gain the estimator was designed by the choice of $V$ and $W$.

$$
\begin{aligned}
& W=\operatorname{diag}(0 \cdot 0002,0 \cdot 001,0 \cdot 001,0 \cdot 001,0 \cdot 001,0 \cdot 001,0 \cdot 001) \\
& V=\operatorname{diag}(1,10,100,10,1)
\end{aligned}
$$

The sampling time, $T=0 \cdot 1 \mathrm{sec}$ and the eigenvalues of the controller and estimator are shown in Fig. 5.

The covariances for the variation of the network equivalent parameters are

$$
\operatorname{diag}(0 \cdot 01,0 \cdot 01,0 \cdot 01,0 \cdot 01)
$$

\subsection{Simulation of dynamics}

In this section, we shall give some simulation results where the control system is working against a simulator of the power system shown in Fig. 6. The simulator is realized by a computer program. Figure 7 shows the results from

$$
\begin{aligned}
& t=6.0 \mathrm{sec}:-0.08-j 0.04 \text { load change at bus } 2 \\
& t=12.0 \mathrm{sec}: \quad 0.08+j 0.04 \text { load change at bus } 2
\end{aligned}
$$

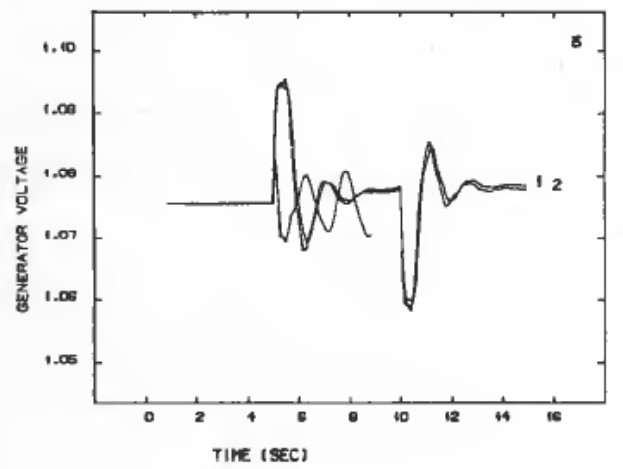

(a) 

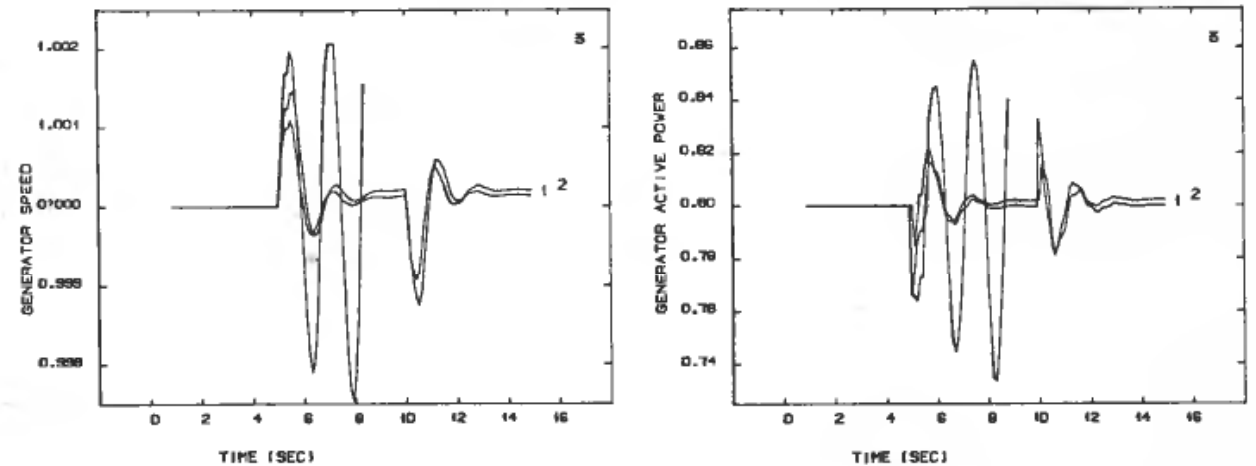

(b)
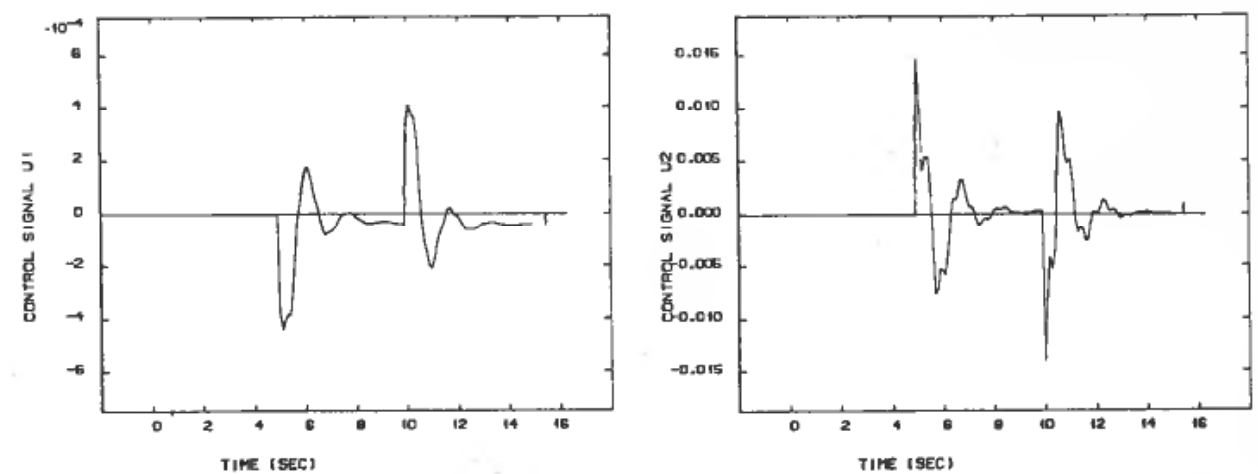

$(d)$
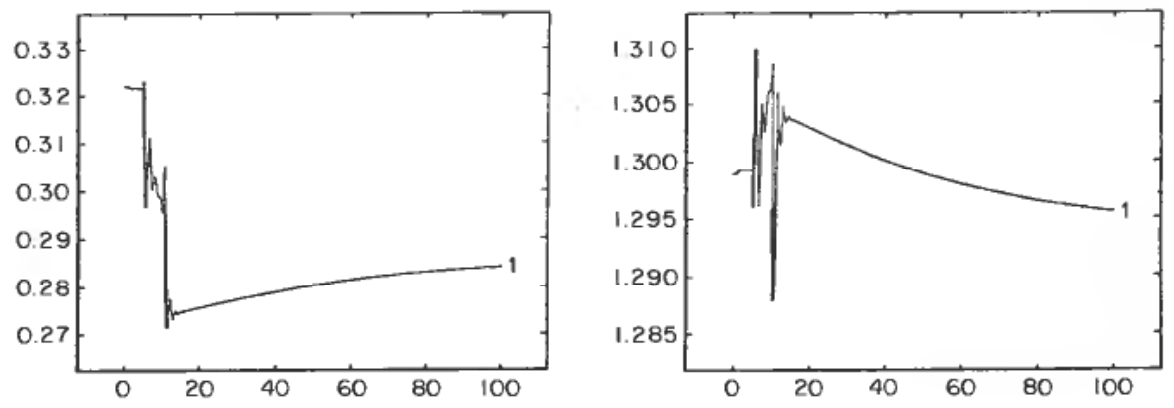

(f)

$(g)$

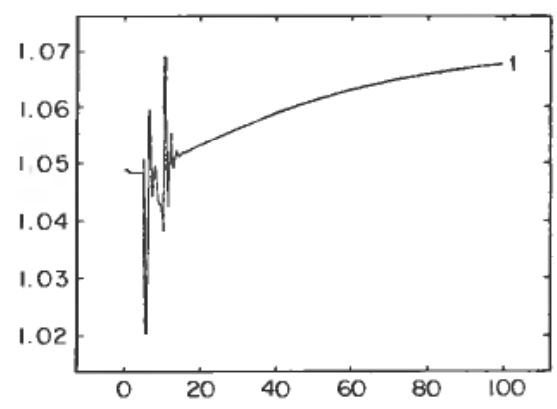

(h)

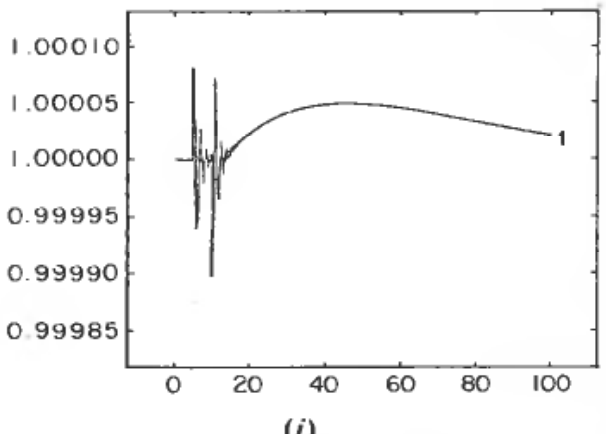

(i)

Figure 7. Simulated value, 1 ; estimated value, 2 ; simulation of machine without controller, 3 for $(a)$ generator voltage, $(b)$ generator active power, $(c)$ generator speed, $(d)$ control signal $u_{1},(e)$ control signal $u_{2},(f)$ estimation of $r,(g)$ estimation of $x,(h)$ estimation of $U,(i)$ estimation of $\omega$. 
We observe that both the damping of power oscillations are increased, and the working point controlling is satisfactory. The estimation of the network equivalent parameters seems to converge, though slowly, in 100-200 seconds.

\section{Conclusion}

Implementing the control system demands a large computing capacity. The augmented Kalman filter, especially, is time consuming. Simulations have shown that the algorithm used approx. $300-400 \mathrm{msec}$ of computing time on a NORD 10 computer, and should be less than 100. If implementing an adaptive multivariable control system on a hydroelectric generating unit, there is therefore a need to reduce the computing time of the algorithm to justify the cost of necessary computer installation. However, simulations have shown that damping of power oscillations are increased, and the adapting algorithm of the optimal controller gives better damping with great changes in operating conditions. The performance of the working point controller is also satisfactory.

\section{Appendix}

The elements of the linearized model are shown in the following.

Matrix A

$$
\begin{aligned}
& a_{12}^{\prime}=100 \pi \\
& a_{22}^{\prime}=\left(\alpha_{2}-D\right) / T_{a} \\
& a_{23}^{\prime}=-I_{q} / T_{a} \\
& a_{24}^{\prime}=\alpha_{3} / T_{a} \\
& a_{33}^{\prime}=-1 / T_{d 0}^{\prime} \\
& a_{44}^{\prime}=-1 / T_{y}
\end{aligned}
$$

$\alpha_{1}, \alpha_{2}, \alpha_{3}$ are linearized from the turbine efficiency and turbine model.

$$
\begin{aligned}
& \alpha_{1}=\frac{\partial m t}{\partial h} \\
& \alpha_{2}=\frac{\partial m t}{\partial \omega g} \\
& \alpha_{3}=\frac{\partial m t}{\partial \gamma}
\end{aligned}
$$

Matrix $B$

$$
\begin{aligned}
& b_{32}=K e / T_{d 0}^{\prime} \\
& b_{41}=K_{y} / T_{y}
\end{aligned}
$$

Matrix $E$

$$
\begin{aligned}
& e_{21}=-\left(x_{q}-x_{d}^{\prime}\right) I_{q} / T_{a} \\
& e_{22}=-\left(E_{q}^{\prime}{ }_{q}+\left(x_{q}-x_{d}^{\prime}\right) I_{d}\right) / T_{a} \\
& e_{31}=-\left(x_{d}-x_{d}^{\prime}\right) / T_{d 0}^{\prime}
\end{aligned}
$$


Matrix $D$

$$
\begin{aligned}
& d_{12}^{\prime}=1 \\
& d^{\prime}{ }_{23}=U_{q} / U_{g} \\
& d_{41}^{\prime}=Q_{g} \\
& d_{43}^{\prime}=U_{q} P_{g} / U_{g}{ }^{2} \\
& d_{51}^{\prime}=-P_{g} \\
& d^{\prime}{ }_{53}=U_{g} Q_{g} / U_{g}^{2} \\
& d_{64}^{\prime}=1 \\
& d^{\prime}{ }_{75}=1
\end{aligned}
$$

Matrix $F$

$$
\begin{aligned}
& f_{21}=-x_{d}^{\prime} U_{g} / U_{g} \\
& f_{22}=x_{q} U_{d} / U_{g} \\
& f_{31}=I_{d} / I_{g} \\
& f_{32}=I_{q} / I_{g} \\
& f_{41}=U_{g} \sin \gamma-x_{d}^{\prime} P_{g} U_{q} / U_{g}{ }^{2} \\
& f_{42}=U_{g} \cos \gamma+x_{q} P_{g} U_{d} / U_{g}{ }^{2} \\
& f_{51}=U_{g} \cos \gamma-x_{d}^{\prime} Q_{g} U_{g} / U_{h g}{ }^{2} \\
& f_{52}=-U_{g} \sin \gamma+x_{q} Q_{g} U_{d} / U_{g}{ }^{2}
\end{aligned}
$$

Matrix $H$

$$
\begin{aligned}
h_{11} & =U\left(\sin \gamma-r \cos \gamma / x_{2}\right) / x_{1} \\
h_{13} & =1 / x_{1} \\
h_{21} & =U\left(\left(1-r^{2} / x_{1} x_{2}\right)\left(\cos \gamma+r \sin \gamma / x_{1}\right) / x_{2}\right. \\
h_{23} & =r / x_{1} x_{2} \\
x_{1} & =x+x_{d}+r^{2} / x_{2} \\
x_{2} & =x+x_{q} \\
U_{d} & =X_{q} I_{q} \\
U_{q} & =E^{\prime}{ }_{q}-X^{\prime}{ }_{d} I_{d}
\end{aligned}
$$

For the calculation of the water turbine efficiency the turbine torque equation is linearized around a working point

$$
m_{t}=m_{t 0}+\alpha_{1} \Delta h+\alpha_{2} \Delta \omega_{g}+\alpha_{3} \Delta y
$$

and the deviation in turbine flow is

$$
q_{t}=q_{t o}+\beta_{1} \Delta h+\beta_{2} \Delta \omega_{s}+\beta_{3} \Delta y
$$



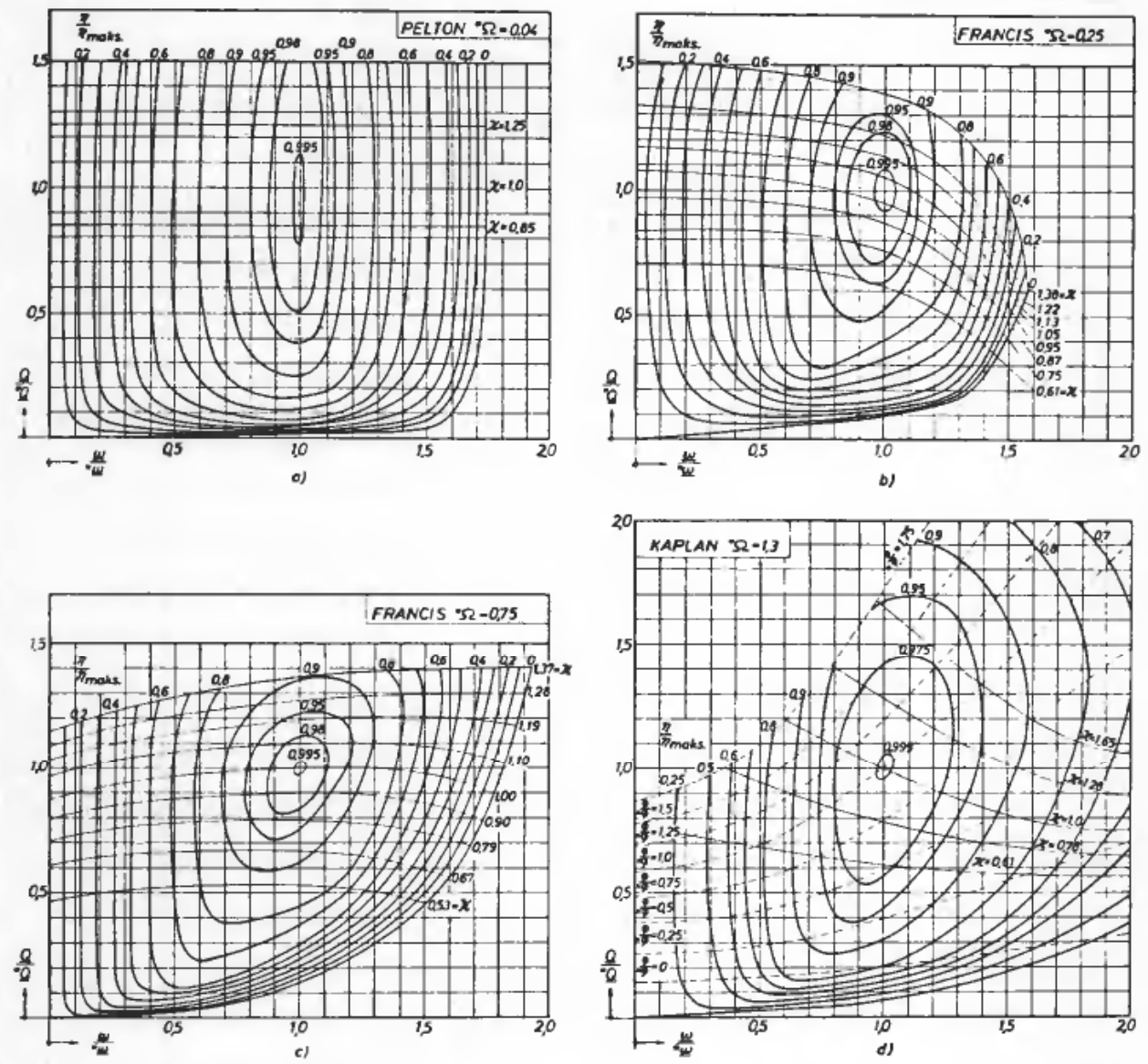

Figure Al. Efficiency diagrams for 4 water turbines. The efficiency is expressed by 'reduced' p.u. shaft speed and turbine flow.

The figure shows diagrams for some water turbines. The turbine efficiency is expressed by reduced per unit shaft speed and turbine water flow, $\omega_{g}$ and $\boldsymbol{q}_{t}$.

$$
\begin{aligned}
& \omega_{g}=\frac{\omega_{g}}{\sqrt{ } h} \\
& q_{t}=\frac{q_{t}}{\sqrt{ } h}
\end{aligned}
$$

In the diagram the maximum efficiency is $1 \cdot 0$, and the fact that the maximum efficiency is somewhat 0.93 is taken into account when calculating the p.u. turbine torque.

$$
m_{t}=\frac{\eta}{{ }^{*} \eta} q h \frac{1}{\omega_{g}}=\eta^{\prime} \frac{q h}{\omega_{g}}
$$

where $\eta=$ true turbine efficiency and ${ }^{*} \eta=$ true maximum efficiency.

The efficiency diagram of the actual turbine is approximated mathematically by

$$
\eta^{\prime}=\eta^{\prime}\left(\omega_{g}, q_{t}\right) \approx \theta_{1} \omega_{g}^{2}+\theta_{2} q_{t}^{2}+\theta_{3} \omega_{g} q_{t}+\theta_{4} \omega_{g}+\theta_{5} q_{t}+\theta_{6}
$$


The constants of the previous equations are found by a least square technique from a collection of points in the actual working area in the efficiency diagram of the turbine. The equations of the turbine flow and torque can now be expressed

$$
\begin{gathered}
q_{t}=\xi_{1} y \omega_{g}+\xi_{1} \omega_{g}+\xi_{3} y+\xi_{4} \\
\beta_{1}=\frac{\partial q_{t}}{\partial h}=\frac{1}{2 \sqrt{ } h} q_{t} \\
\beta_{2}=\frac{\partial q_{t}}{\partial \omega_{g}}=\frac{\partial q_{t}}{\partial \omega_{g}}=\xi_{1} y+\xi_{2} \\
\beta_{3}=\frac{\partial q_{t}}{\partial y}=\sqrt{ } h \frac{\partial q_{t}}{\partial y} \\
\frac{\partial q_{t}}{\partial y}=\xi_{1} \omega_{g}+\xi_{3} \\
\frac{\partial \eta^{\prime}}{\partial h}=0 \\
\frac{\partial \eta^{\prime}}{\partial \omega_{g}}=\frac{\partial \eta^{\prime}\left(q_{t}, \omega_{g}\right)}{\partial \omega_{g}}+\frac{\partial \eta^{\prime}\left(q_{t}, \omega_{g}\right)}{\partial q_{t}} \cdot \frac{\partial q_{t}\left(y, \omega_{g}\right)}{\partial \omega_{g}} \\
\frac{\partial \eta^{\prime}\left(q_{t}, \omega_{g}\right)}{\partial \omega_{g}}=2 \theta_{1} \omega_{g}+\theta_{3} q_{t}+\theta_{4} \\
\frac{\partial \eta^{\prime}\left(q_{t}, \omega_{g}\right)}{\partial q_{t}}=2 \theta_{2} q_{t}+\theta_{3} \omega_{g}+\theta_{5} \\
\frac{\partial \eta^{\prime}}{\partial y}=\frac{\partial \eta^{\prime}\left(q_{t}, \omega_{g}\right)}{\partial q_{t}} \cdot \frac{\partial q_{t}}{\partial y} \\
m_{t}=\eta^{\prime} h q / \omega_{g} \\
\alpha_{1}=\frac{\partial m_{t}}{\partial h}=\frac{\eta^{\prime}}{\omega_{g}} \frac{q_{t}}{\omega_{g}}=\eta_{\eta^{\prime}} \frac{q_{t}}{\omega_{g} y}\left(\eta^{\prime} q_{t}\right) \\
\omega_{g}
\end{gathered}
$$


The following tables show data for the network in Fig. 6.

\begin{tabular}{llrrr}
\hline Bus & Voltage & Angle & \multicolumn{1}{l}{$\begin{array}{l}\text { Active } \\
\text { power }\end{array}$} & \multicolumn{1}{c}{$\begin{array}{c}\text { Reactive } \\
\text { power }\end{array}$} \\
\hline 1 & 1.0 & 0 & $-0 \cdot 345$ & $0 \cdot 134$ \\
2 & 1.0 & 31 & $-0 \cdot 600$ & $-0 \cdot 200$ \\
3 & 1.076 & 38 & $0 \cdot 800$ & $0 \cdot 240$ \\
4 & 1.061 & 35 & $0 \cdot 200$ & $0 \cdot 160$ \\
\hline
\end{tabular}

Table A 1. Voltages and powers for the busbars.

\begin{tabular}{lll}
\hline Line & Resistance & Reactance \\
\hline $1-2$ & $0 \cdot 144$ & $1 \cdot 44$ \\
$2-3$ & $0 \cdot 057$ & $0 \cdot 22$ \\
$2-4$ & $0 \cdot 072$ & $0 \cdot 29$ \\
$3-4$ & $0 \cdot 144$ & $0 \cdot 72$ \\
\hline
\end{tabular}

Table A 2. Line impedances.

\begin{tabular}{|c|c|c|c|c|c|c|c|c|}
\hline \multirow[b]{3}{*}{ Bus } & \multicolumn{8}{|c|}{ Generators } \\
\hline & \multicolumn{5}{|c|}{ Reactances } & \multicolumn{3}{|c|}{ Time constants } \\
\hline & $T_{a}$ & $x_{d}$ & $x_{d}^{\prime}$ & $x_{q}$ & $x^{\prime \prime}{ }_{d}$ & $T_{d 0}^{\prime}$ & $T_{d 0}^{\prime \prime}$ & $T^{\prime \prime}{ }_{q 0}$ \\
\hline 1 & 2400 & 0.25 & $0 \cdot 08$ & $0 \cdot 15$ & 0.06 & $6 \cdot 0$ & $0 \cdot 05$ & $0 \cdot 15$ \\
\hline 3 & 6 & $1 \cdot 20$ & 0.30 , & 0.75 & 0.26 & $7 \cdot 5$ & 0.05 & $0 \cdot 15$ \\
\hline 4 & $1 \cdot 2$ & $2 \cdot 87$ & 0.62 & $1 \cdot 75$ & 0.55 & $6 \cdot 0$ & 0.05 & $0 \cdot 15$ \\
\hline
\end{tabular}

Table A 3. Data for generators.

The following shows the data for the model of the generator at bus 3 .

$$
\begin{array}{rlrl}
\alpha_{2} & =-1,0 & K_{E} & =100,0 \\
D & =5,0 & K_{y} & =1,0 \\
\alpha_{3} & =1,0 & x_{d} & =1,2 \\
\alpha_{1} & =0,7 & x_{q} & =0,75 \\
T^{\prime}{ }_{d 0} & =7,5 & x_{d}^{\prime} & =0,3 \\
T_{\gamma} & =0,3 & r & =0,323 \\
U & =1,05 & x & =1,3 \\
T & =0,1 & \omega & =1,0
\end{array}
$$

All data is given relative a $132 \mathrm{kV}, 250 \mathrm{MVA}$ base. 


\section{REFERENCES}

BREKKE, H., and BUGAARDEN, K. (1980). A study on governing problems caused by transients in high pressure tunnel systems. IAHR, AIRH. Symposium I980. Tokyo.

Habibullah, B., and YU, Y. (1974). Physically realizable wide range optimal controllers for an electric power system. I.E.E.E. Power apparatus and systems T.-74, 120-2.

HEGGLID, G. J. (1981). Multivariable regulator for vannkraftaggregater. Norwegian Institute of Technology. Ph.D. Thesis in norwegian.

IBRAHIM, M. (1976). Dynamic equivalents using operating data and stochastic modelling. I.E.E.E. Power apparatus and systems 95.

Khobragade, N. T. (1979). Synthesis of optimal controller for synchronous power system. Electric Power Research (vol. 2).

KhobraGADE, N. T., and TAMSKAR, D. G. (1979). Optimal controller for synchronous power system with prescribed degree of stability. Electric Power Research (vol. 2).

PRICE, W. W., et al. (1974). Maximum likelihood identification of power system dynamic equivalents. I.E.E.E. Conference on Decision and Control, Phoenix, Arisona.

PRicE, W. W., et al. (1975). Dynamic equivalents from on-line measurements. I.E.E.E. Power apparatus and systems 94.

RuDNICH, H., et al. (1976). Identification of powers ystem dynamic equivalents. I.E.E.E. Power apparatus and systems 95.

TIWARI, R. N., and TIWARI, S. N. (1979). Evaluating alternative controllers for an electric power system. Electric Power Research (vol. 2).

YU, Y., et al. (1979). Estimation of unknown large power system dynamics. I.E.E.E. Power apparatus and systems 98. 